\title{
Parásitos en avestruces en el Departamento del Meta
}

\section{Parasites in ostriches in the department of Meta}

\author{
Lozada $\mathrm{H}^{1}$, Rincón $\mathrm{J}^{2}$ y López $\mathrm{V}^{2}$ \\ ${ }^{1} \mathrm{MVZ}$, Profesor, Universidad de los Llanos \\ ²icenciadas en Producción Agropecuaria. UNILLANOS \\ hdolozada@yahoo.com.ar
}

Recibido 5 de Marzo 2011, aceptado 4 de Mayo 2011

\section{RESUMEN}

La zoocría de avestruces es una de las nuevas alternativas productivas presentes en Colombia desde el año 2004; no obstante, son escasos los antecedentes sobre el manejo en cautiverio y faltan estudios acerca de las enfermedades parasitarias que estas aves pueden padecer en las condiciones del Pie de Monte Llanero. A partir de esa situación, dos aspirantes al título de Licenciatura en Producción Agropecuaria, de la Universidad de los Llanos y un profesor Médico Veterinario y zootecnista, diseñaron y ejecutaron un trabajo de investigación, utilizando animales de un zoocriadero localizado sobre el kilómetro doce (12) de la vía a Puerto López, que conduce al municipio de Puerto Gaitán, en el Departamento del Meta, donde se encuentra la vereda "Alto navajas", donde se localiza la finca "El desquite" y dentro de éste, el zoocriadero "Latin American". Allí se conformaron grupos etarios para la toma y posterior procesamiento técnico en el laboratorio, de muestras coprológicas, sanguíneas y cutáneas, para buscar parásitos. Se tomaron cuatro (4) muestras con intervalos de 45 días. Se detectaron cuatro (4) géneros de parásitos, de los cuales tres (3) corresponden a los internos conocidos como: Ascaridia galli ( $A$. G.), Entamoeba $s p$ (E. Sp.) y Libyostrongylus $s p$, (L. Sp.) en tanto que el parásito externo que se identificó fue Strupthiopterolichus sculpturatus (S. S.), ácaro de las plumas.

Palabras clave: Rátidas, ácaros, nematodos. 


\begin{abstract}
The ostriches breeding is one of the new alternatives productive present in Colombia (South America), since 2004; nevertheless, there are scarce the local antecedents on the handling in captivity and they lack studies about the parasitic illnesses that these birds can suffer under the conditions of the eastern Plains of Colombia. Starting from that situation, two students of Degree in Agricultural Production and a Veterinary Medical professor into Eastern plains University, designed and they executed an investigation work, using animals of a farm located on the twelve (12) kilometer of the road that of the municipality of "Puerto López", going to the municipality of "Puerto Gaitán", on Meta Department, where it is the veredal zone "Alto navajas", there the property "EI desquite" is located, and into it, the "Latin American" zoo farm. There they integrated by groups of different ages, for the taking and later technical process in the laboratory, of samples of blood, skin and fecal matter, to looking for parasites. Four (4) samplings were made with intervals of 45 days between them. Four (4) genres of parasitic were detected; of those which three (3) are the well-known internal parasites as Ascaridia galli, Entamoeba $s p$ and Libyostrongylus $s p$ while the external parasite called Strupthiopterolichus sculpturatus were identified, the feathers acarus.
\end{abstract}

Keywords: Ratites, mites, nematodes.

\title{
INTRODUCCIÓN
}

El avestruz pertenece al grupo de las rátidas debido a que no puede volar, tienen bien desarrolladas y fuertes sus piernas, es el ave más grande que ha logrado adaptarse a una vida terrestre. Esta ave habita de forma silvestre en África y en Australia. Los cazadores codician su carne y plumas causando su extinción en varios sitios. Para conservar esta especie, fundaron instituciones en Sudáfrica y Australia, donde existen los mayores criaderos. Como consecuencia de esto se generaron varias razas domésticas, que son explotadas de forma productiva, no solo para carne, sino que la piel (cuero) se utiliza para la fabricación zapatos (Jiménez, 2003). 
El macho es más grande que la hembra, mide 2.7 metros de altura y pesa 145 $\mathrm{Kg}$, además tiene las plumas del cuerpo negras y las del borde de las alas y la cola blancas, mientras las hembras son gris opaco, presentando menos plumas blancas en sus alas, puede medir 2.3 metros y pesar 110 kilos (Figura 1). Los avestruces pueden correr a una velocidad de 60 a $70 \mathrm{~km} / \mathrm{h}$. Los huevos son blancos y grandes, pesando alrededor de $1.6 \mathrm{Kg}$ (Jiménez, 2003).

Los parásitos son un problema sanitario que afecta a las aves siendo las más frecuentes las infecciones subclínicas que causan la muerte, además, interfieren en su aspecto productivo y reproductivo, las aves hospedan varias especies de parásitos, como: Trichomonas gallinae, Trichostrongylus tenuis, Ascaridia columbae, A. sprenti, A. dissimilis, Heterakis gallinarum, Capillaria sp, Eimeria dispersa y Raillietina sp, entre otros (Figueiro et al., 2002).

En Colombia la zoocría de avestruces (Struthio camellus) es una de las nuevas alternativas productivas en el país desde el año 2004, pese a esto son escasos los antecedentes sobre el manejo en cautiverio y estudios de enfermedades parasitarias, de manera que la mayoría de ellas y muertes observadas ocurren por la presencia de cuerpos extraños, problemas nutricionales y manejos sanitarios inadecuados. Es de prever que, con el desarrollo de la zoocría de esta especie se va a generar un medio propicio para que surjan nuevas enfermedades o que sean transmitidas por otros animales. Por este motivo, la existencia de instalaciones de cría, o comercialización de productos de estas aves, puede suponer un potencial paso de parásitos y otros problemas de ciclos en animales silvestres que pasen a los domésticos y al hombre.

Con estos antecedentes se entendió que era posible el estudio de la epidemiología parasitaria de esta especie, realizado en el zoocriadero "Latin American" por lo tanto se hizo necesario realizar pruebas para endo y ecto parásitos en los avestruces. De acuerdo a los estudios los objetivos de esta investigación fueron: recolección, clasificación de parásitos hasta nivel de género y determinación del grado de infestación de los especímenes hallados en los animales. 


\section{MATERIALES Y MÉTODOS}

La investigación se realizó en el zoocriadero "Latín American", ubicado en el kilómetro doce (12) de la vía que de Puerto López conduce a Municipio de Puerto Gaitán, en el Departamento del Meta, tomando el desvío a mano derecha, para dirigirse a la vereda "Alto Navajas", donde se localiza la finca "EI Desquite", con una altitud de 400 m.s.n.m., temperatura de $32^{\circ} \mathrm{C}$ y precipitación anual entre 1800 y $3000 \mathrm{~mm}$. Se colectaron muestras de sangre y materia fecal, en tres (3) grupos etarios, para buscar los posibles géneros parasitarios que pudieran estar afectándolos.

Se trabajó con cuarenta y cinco (45) animales divididos en tres (3) grupos etarios de quince ejemplares cada uno. La toma de muestras se realizó al $33,33 \%$ de la población total, tomando cinco (5) individuos de cada grupo en forma aleatoria. Estas muestras fueron recolectadas con intervalos de cuarenta y cinco (45) días, teniendo en cuenta el ciclo parasitario, entre los meses de Febrero y Junio del año 2010, para un total de cuatro (4) muestreos en el zoocriadero.

Figura 1. Trío de avestruces, dos hembras un macho

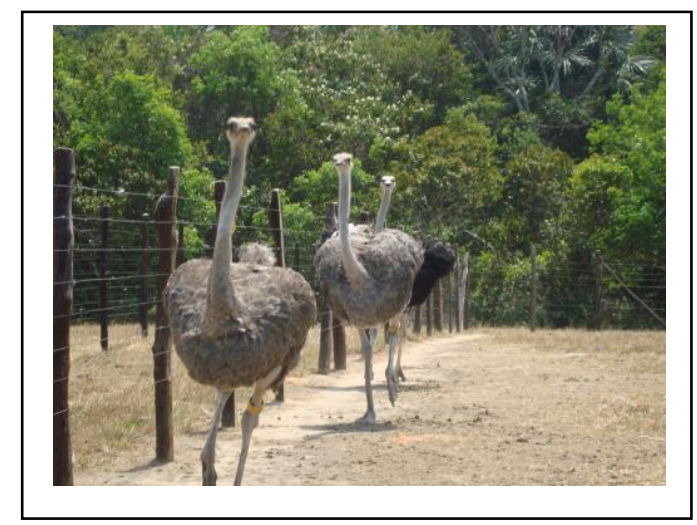

Las muestras fueron analizadas en un laboratorio Especializado y en el laboratorio de Parasitología Animal de la Universidad de los Llanos, con el fin de determinar los géneros hallados. Las muestras fecales se recolectaron de avestruces, directamente de la zona cloacal, evitando la contaminación del suelo; éstas fueron transportadas en bolsas plásticas y puestas en refrigeración hasta el momento de su análisis (Figura 2). 


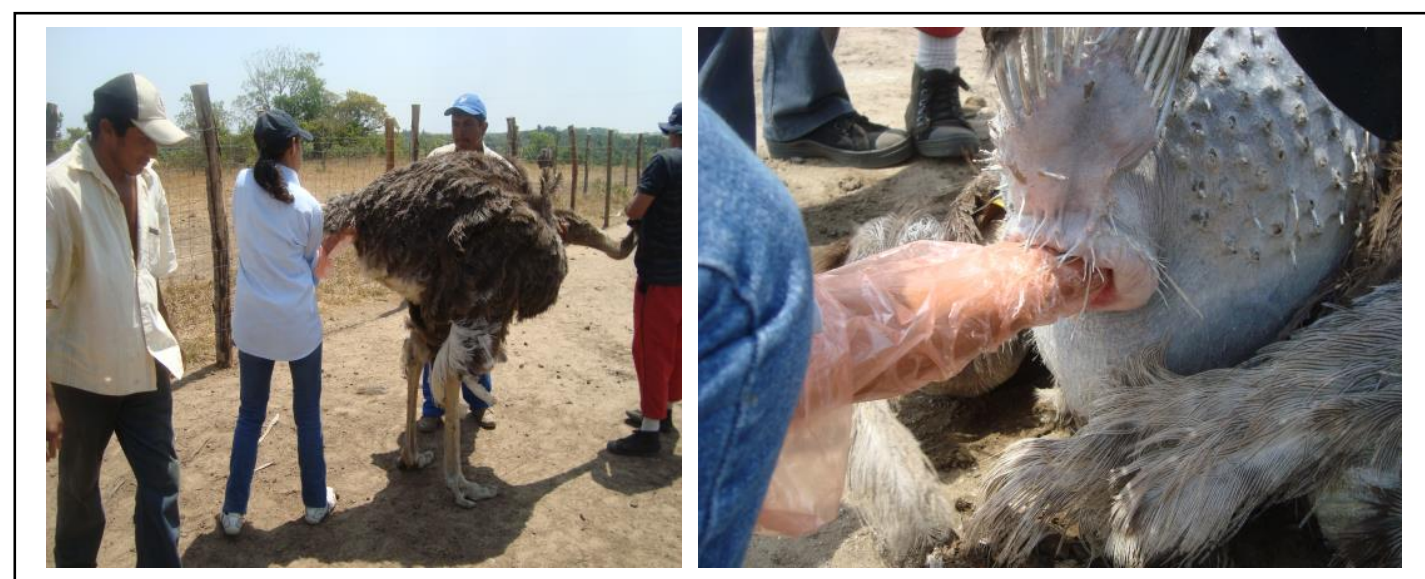

Figura 2. Toma de muestra fecal directamente de la zona cloacal

Para la coprología se utilizó la técnica de Gloss, revisada por Basso et al., (1998) y la de Mc Máster, revisada por Margolis et al., (1982) trabajando con muestras comunitarias en cada grupo etario. Para detectar la presencia de hemoparásitos, se utilizó el frotis sanguíneo y la técnica de Woo, revisado por Quispe et al., (2003) (Figura 3).

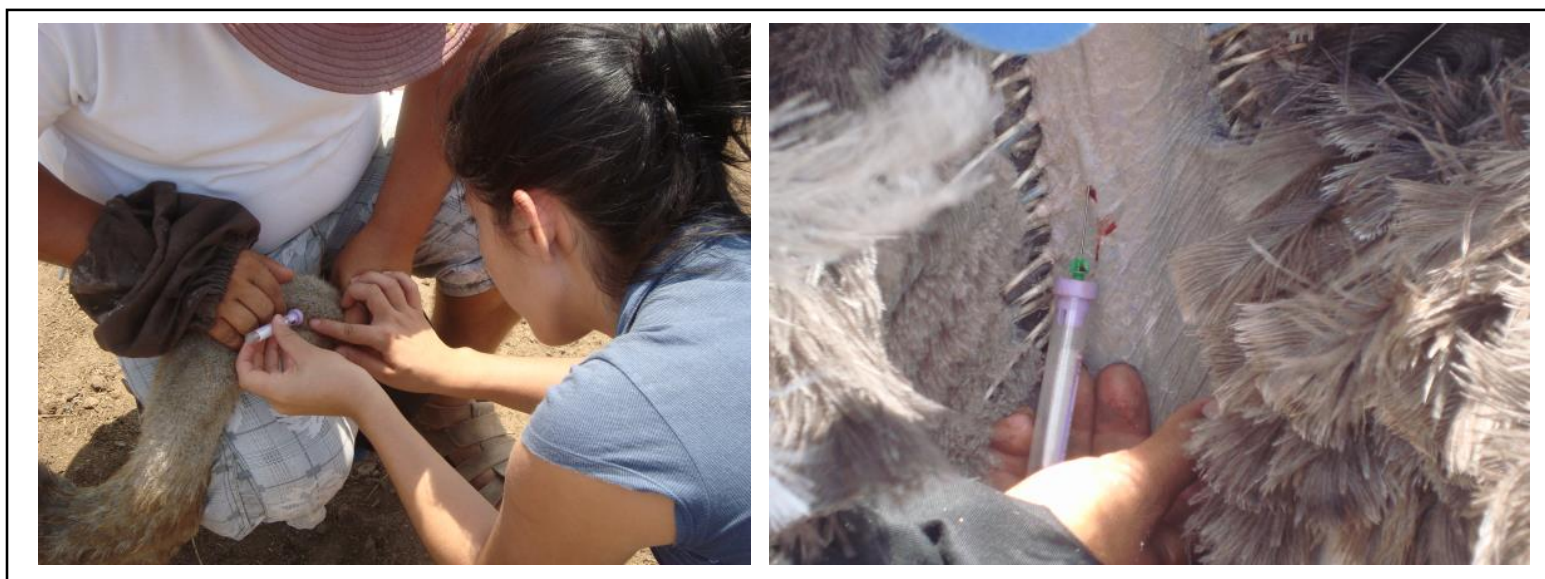

Figura 3. Toma de sangre en las venas alar y yugular

Para el caso de ectoparásitos, las muestras se colocaron en bolsas plásticas identificadas con el grupo etario correspondiente. Los ectoparásitos fueron recogidos con una pinza anatómica lisa, fijados y conservados en alcohol de $70^{\circ}$. Los parásitos se fijaron entre porta y cubre objetos, utilizando resina sintética "Entellen", para su posterior identificación. Se empleó un sistema de fotografía ACT-2U, acoplado a una cámara de fotografía y a un microscopio de contraste de fase, eclipse E 400, perteneciente al grupo de investigación de 
toxicología del Instituto de Acuicultura de los Llanos (IALL), donde finalmente los ejemplares se observaron, midieron y se fotografiaron, para poder clasificarlos taxonomicamente con posterioridad.

\section{RESULTADOS Y DISCUSIÓN}

Dentro de la investigación realizada en el zoocriadero "Latín American", se evidenció la presencia de tres (3) parásitos internos identificados como Ascaridia galli, Entamoeba $s p$ y Libyostrongylus sp. Igualmente se detectó un parasito externo conocido fuera del país como acaro chupador de las plumas de avestruces y clasificado como Struthiopterolichus sculpturatus. Los resultados encontrados en los diferentes muestreos se indican en las Tablas 1 , 2, 3, y 4 .

La primera toma de datos, se realizó el 11 de Febrero del 2010, se observó una alta incidencia del parasito externo Struthiopterolichus sculpturatus, ácaro detritívoro en todos los grupos etarios, con un grado de infestación de +++. Dentro del grupo etario de pollos, se encontró un nematodo denominado Ascaridia galli con un grado de infestación de ++ y finalmente la presencia de un protozoario llamado Entamoeba sp en dos de los grupos etarios: Pollos y reproductores, con un grado de infestación de + según el método de Mc Máster (Tabla1).

Tabla 1. Grado de infestación de los parásitos hallados durante el primer muestreo al zoocriadero "Latín American"

\begin{tabular}{lcccc}
\hline \multicolumn{1}{c}{$\begin{array}{c}\text { Grupo } \\
\text { Etario }\end{array}$} & $\begin{array}{c}\text { Struthiopterolichus } \\
\text { sculpturatus }\end{array}$ & $\begin{array}{c}\text { Libyostrongylus } \\
\boldsymbol{s p}\end{array}$ & $\begin{array}{c}\text { Ascaridia } \\
\text { galli }\end{array}$ & $\begin{array}{c}\text { Entamoeba } \\
\boldsymbol{s p}\end{array}$ \\
\hline Pollos & 20 & 0 & 7 & 4 \\
Juveniles & 20 & 0 & 0 & 0 \\
Reproductores & 20 & 0 & 0 & 4 \\
\hline
\end{tabular}

La segunda visita, realizada el día 26 de Marzo de 2010, arrojó como resultado de la investigación, la ausencia de parásitos internos en los tres grupos etarios. Lo anterior se evidencia por que los animales fueron vermífugados un mes antes del presente muestreo con un antihelmíntico de amplio espectro contra parásitos gastrointestinales y pulmonares, en fase de maduración sexual (vermicida), formas juveniles inmaduras y tardías (larvicida) y con acción sobre 
los huevos (ovicida). Cada $1 \mathrm{ml}$ de suspensión acuosa contiene $100 \mathrm{mg}$ de ácido carbámico, (5-benzoil-1H-bencimidazol-2-il) metil éster, como ingrediente activo. La dosificación suministrada a cada animal fue de un (1) ml por animal. La presencia del parásito externo Strupthiopterolichus sculpturatus sigue siendo permanente, con una alta incidencia en los tres grupos etarios. (Tabla 2).

Tabla 2. Grado de infestación del parásito Struthiopterolichus sculpturatus en los tres grupos etarios

\begin{tabular}{lcccc}
\hline $\begin{array}{c}\text { Grupo } \\
\text { Etario }\end{array}$ & $\begin{array}{c}\text { Struthiopterolichus } \\
\text { sculpturatus }\end{array}$ & $\begin{array}{c}\text { Libyostrongylus } \\
\boldsymbol{s p}\end{array}$ & $\begin{array}{c}\text { Ascaridia } \\
\text { galli }\end{array}$ & $\begin{array}{c}\text { Entamoeba } \\
\boldsymbol{s p}\end{array}$ \\
\hline Pollos & 20 & 0 & 0 & 0 \\
Juveniles & 20 & 0 & 0 & 0 \\
Reproductores & 20 & 0 & 0 & 0 \\
\hline
\end{tabular}

El tercer registro fue realizado el 7 de Mayo del 2010, se observó una alta incidencia del parásito externo Strupthiopterolichus sculpturatus, en todos los grupos etarios, con un grado de infestación de: +++. Dentro del grupo etario de juveniles, se encontró un nematodo denominado Ascaridia galli con un grado de infestación de: ++ según el método de Mc Máster. También se observó una alta incidencia del parasito externo S.s., en todos los grupos etarios, con un grado de infestación de: +++. Dentro del grupo etario de juveniles, se encontró un nematodo denominado Ascaridia galli con un grado de infestación de: ++ según el método de Mc Máster (Tabla 3).

Tabla 3. Grado de infestación del parásito Struthiopterolichus sculpturatus en los tres grupos etarios y el nematodo Ascaridia galli_en el grupo de los juveniles

\begin{tabular}{lcccc}
\hline \multicolumn{1}{c}{$\begin{array}{c}\text { Grupo } \\
\text { Etario }\end{array}$} & $\begin{array}{c}\text { Struthiopterolichus } \\
\text { sculpturatus }\end{array}$ & $\begin{array}{c}\text { Libyostrongylus } \\
\text { sp }\end{array}$ & $\begin{array}{c}\text { Ascaridia } \\
\text { galli }\end{array}$ & $\begin{array}{c}\text { Entamoeba } \\
\boldsymbol{s p}\end{array}$ \\
\hline Pollos & 20 & 0 & 0 & 0 \\
Juveniles & 20 & 0 & 4 & 0 \\
Reproductores & 20 & 0 & 0 & 0 \\
\hline
\end{tabular}

La cuarta jornada de toma de datos, fue realizada el 21 de Junio de 2010, se obtuvo como resultado de la investigación, la identificación de un parasito especifico de los avestruces y con pocos reportes, el nematodo llamado Libyostrongylus $s p$ comúnmente llamado gusano alambre. Éste se encontró en 
el grupo etario de los juveniles siendo su principal hospedero con un grado de infestación de +; de igual manera se encontró la presencia continua del acaro de las plumas Strupthiopterolichus sculpturatus con una alta infestación de ++++. (Tabla 4).

Tabla 4. Grado de Infestación del parásito Struthiopterolichus sculpturatus en los tres grupos etarios y el nematodo Libyostrongylus $s p$ en el grupo de los juveniles

\begin{tabular}{lcccc}
\hline \multicolumn{1}{c}{ Grupo } & $\begin{array}{c}\text { Struthiopterolichus } \\
\text { Eculpturatus }\end{array}$ & $\begin{array}{c}\text { Libyostrongylus } \\
\text { Ep }\end{array}$ & $\begin{array}{c}\text { Ascaridia } \\
\text { galli }\end{array}$ & $\begin{array}{c}\text { Entamoeba } \\
\boldsymbol{s p}\end{array}$ \\
Pollos & 20 & 0 & 0 & 0 \\
Juveniles & 20 & 4 & 0 & 0 \\
Reproductores & 20 & 0 & 0 & 0 \\
\hline
\end{tabular}

Después del análisis realizado a cada grupo etario teniendo en cuenta la presencia de parásitos internos y externos, se determinó la prevalencia de los géneros parasitarios encontrados en avestruces (Gráfico1).

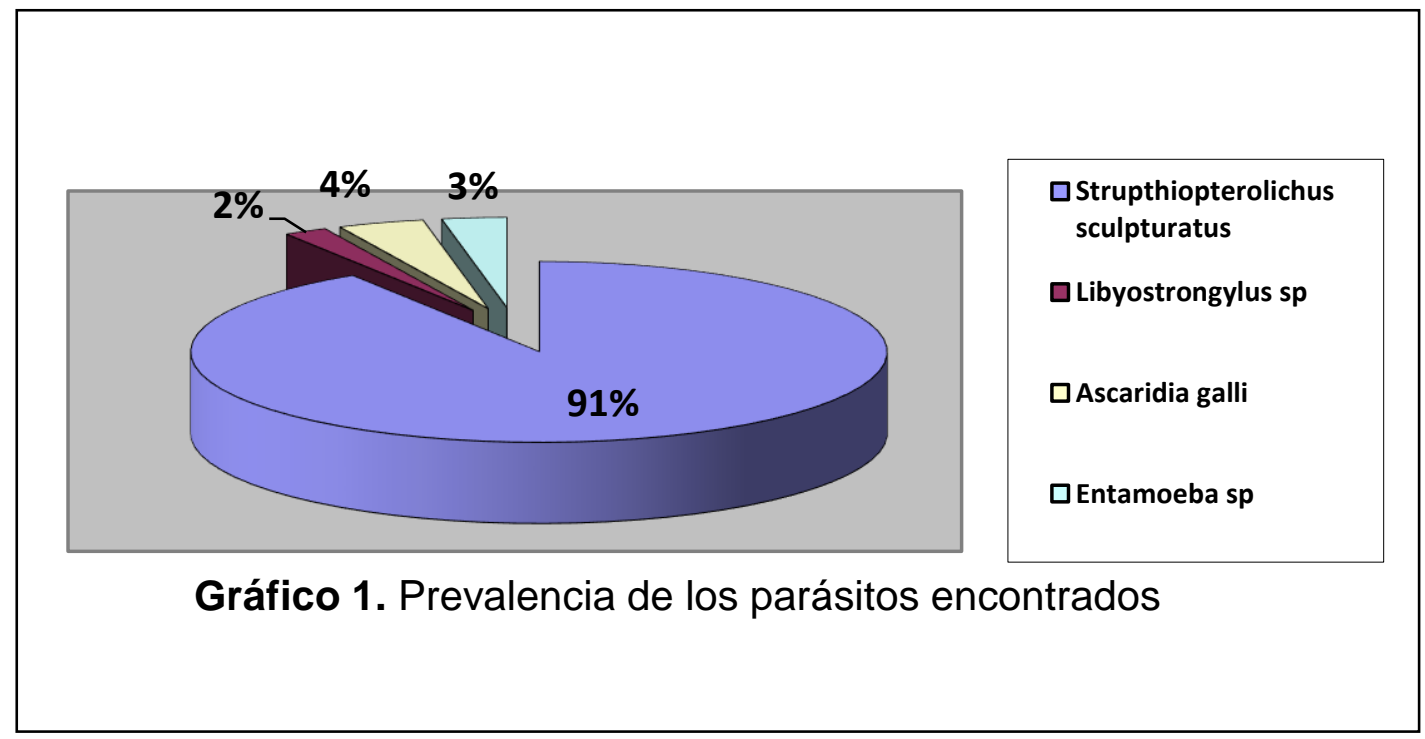

El $2 \%$ de la prevalencia corresponde al nematodo Libyostrongylus $s p$ debido a un grado de infestación de $+(2-4)$ escasas para el grado de infestación en los avestruces. El $4 \%$ de la prevalencia hallada, fue para el nematodo Ascaridia galli, su grado de infestación de ++ (5-7); moderadas para el grado de infestación en los avestruces. El 3\% de la prevalencia fue para el protozoario Entamoeba sp, su grado de infestación es de + (2-4); escasa para el grado de infestación en los avestruces. El 91\% de la prevalencia le correspondió al ácaro 
Strupthiopterolichus Sculpturatus, con un grado de infestación de ++++ (más de 9); muy abundantes (Gráfico 1).

Se tomó como referencia el número veinte (20) para un porcentaje total del 100\% para el grado de infestación de los parásitos hallados en la investigación: Struthiopterolichus sculpturatus: con un porcentaje de infestación del 100\% durante los cuatro (4) muestreos, en los tres grupos etarios. Ascaridia galli: con porcentaje de infestación del $35 \%$ que corresponde a siete (7) dentro del rango planteado. Hallado en el grupo de pollos, y con un $20 \%$ correspondiente a cuatro (4) para los juveniles. Entamoeba sp: con un porcentaje de infestación del $20 \%$ que corresponde a cuatro (4) para pollos y $20 \%$ para reproductores. Libyostrongylus sp: con un porcentaje de infestación del $20 \%$ que corresponde a cuatro (4) para juveniles (Gráfico 2).
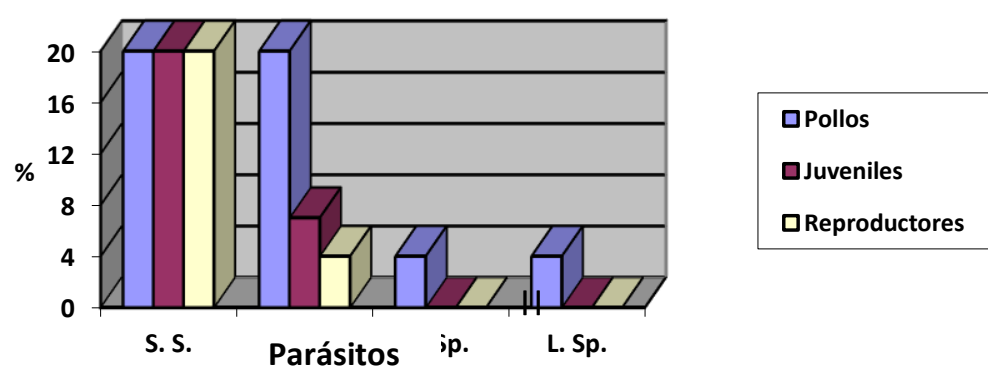

Gráfico 2. Grado de infestación por grupo etario

Es importante señalar que la dinámica pluviométrica en el municipio de Puerto López (Meta), durante la toma de muestras en el sector donde se localiza el zoocriadero "Latín American" influyó de manera significativa en los ciclos parasitarios de los especímenes hallados, se evidencia que durante los días previos a la toma de las tres primeras muestras, (Febrero, Marzo y Mayo), hubo predominio de tiempo seco en el territorio donde se localiza el zoocriadero, las lluvias se presentaron de manera importante en el mes de junio.

Al correlacionar la variable pluviométrica con los resultados de los muestreos, se observa que la presencia del ácaro Struthiopterolichus sculpturatus, es independiente, pues está presente de manera constante. La presencia de 
Libyostrongylus $s p$, en el grupo etario juvenil, sí se relaciona con las lluvias abundantes, pues solo se detectó en el último muestreo.

Por otra parte, llama la atención la presencia de Ascaridia galli en el primer muestreo en los pollos; grupo al cual se le suministró un (1) $\mathrm{ml}$ ácido carbámico, (5-benzoil-1H-bencimidazol-2-il) metil éster a cada animal un mes antes del segundo muestreo, arrojando total ausencia en los siguientes resultados. Éste nematodo se presenta nuevamente con irregularidad en el tercer muestreo en el grupo etario de juveniles, debido a que estos animales no fueron vermifugados con anterioridad.

La presencia de Entamoeba sp se registró debido a la poca sanidad en los bebederos utilizados, adicionalmente al hecho de la existencia de aguas estancadas en algunos sectores del zoocriadero.

El grado de infestación de los parásitos internos fue bajo en comparación a la infestación del parásito externo Strupthiopterolichus sculpturatus. Este ácaro fue detectado en un alto porcentaje de individuos; se localiza principalmente en la base de las plumas causando daños a éstas en su raquis, plumón y cálamo. Aunque no se asocia a este parásito externo con la transmisión de enfermedades, el hecho de que ataquen a las plumas puede provocar una pérdida en la calidad de este producto que tiene valor comercial y generar estrés permanente, lo que tiene consecuencias nefastas para la explotación. Se realizo un ensayo con un producto organofosforado, el cual fue completamente inocuo para el parásito. Este ácaro, identificado en el laboratorio de parasitología de la Universidad de los Llanos, fue referenciado por el MVZ Hernando Lozada Monroy, en el transcurso de la presente investigación.

Dada la carencia de datos comprobables por parte de las autoridades sanitarias (ICA), sobre la presencia de este parásito en Colombia, este trabajo se constituye en el primer reporte documentado para los Llanos Orientales. A manera de cortesía profesional se le remitió al centro de diagnóstico veterinario del ICA de Villavicencio una muestra del parásito externo Struthiopterolichus sculpturatus. Adicional a lo anterior se encuentra disponible un tutorial temático en el centro de documentación de la Universidad de los Llanos y en el centro 
de diagnóstico del ICA seccional Meta. Los datos que se tomaron como base documental, proceden del ámbito internacional, que se concentra en países que manejan comercialmente la especie, principalmente Brasil, Chile, España y África.

\section{CONCLUSIONES}

\section{Ascaridia galli}

Gusanos nematodos, blanco-amarillento, los machos 50-76 mm de largo, 116 $\mathrm{mm}$ de largo (Figura 4). Los huevos de 73-92 x $45 \mu \mathrm{m}$, elípticas, lisas gruesa cáscara $57 \mu \mathrm{m}$ (Figura 5), es oval, su cutícula externa es albuminosa, presenta morulaciones de color oscuro y con aspecto granuloso; estas observaciones se hicieron en el Laboratorio de Parasitología Animal de la Universidad de los Llanos en el desarrollo del presente trabajo.

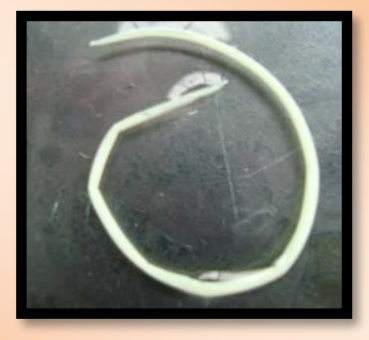

Figura 4. Adulto de Ascaridia galli

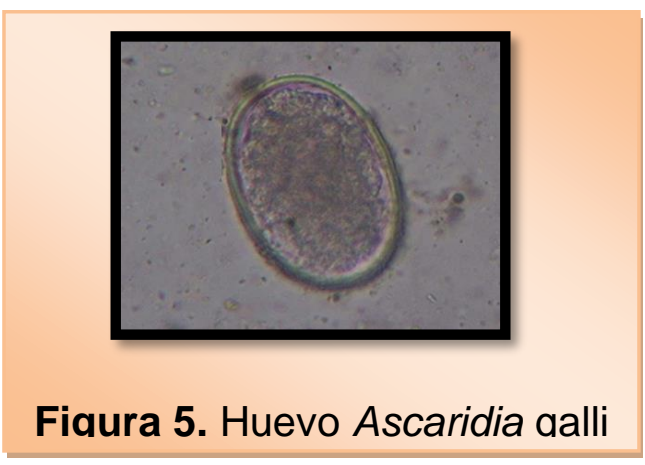

\section{Libyostrongylus sp}

El huevo se caracteriza por su forma ovalada con polos iguales, cutículas gruesas, mórulas bien formadas y de gran tamaño. Observada a $40 \mathrm{x}$ en el laboratorio de Parasitología Animal de la Universidad de los Llanos en el desarrollo del presente trabajo. (Figuras 6, 7 y 8).
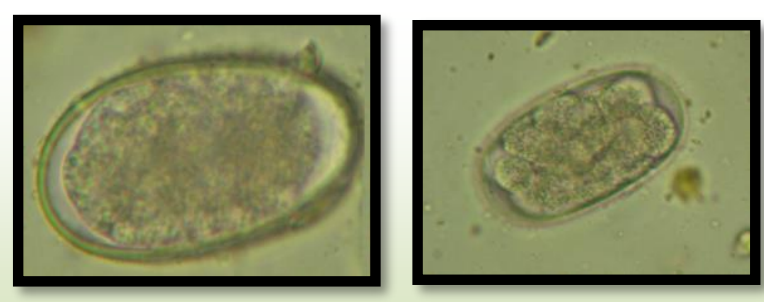

Figura 6. Huevos de Libyostrongylus sp. 

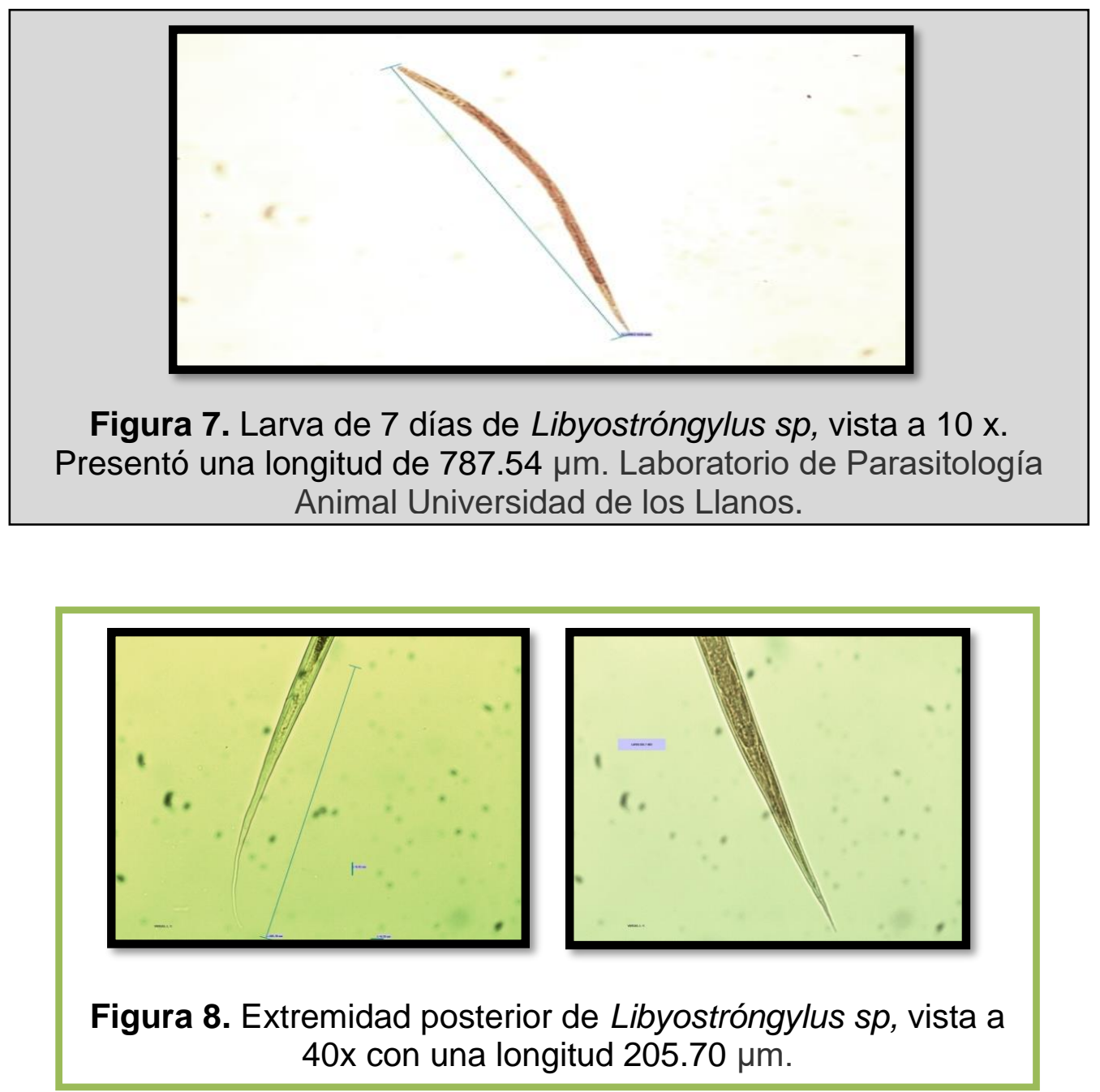

\section{Struthiopterolichus sculpturatus}

Describiendo su morfología tiene cuerpo aplanado dorso-ventral, coloca las piernas lateralmente al cuerpo, ambulacra grande del tarso para ayudar a su apego a las bárbulas de plumas y el cuerpo, corto en su superficie dorsal, por lo general se presenta como así esclerotizado, características validadas para el macho Struthiopterolichus sculpturatus en escala observada en el desarrollo del presente trabajo, con apoyo del grupo GRITOX del IALL. Las dimensiones de ácaro son: cuerpo: longitud: 470,0 y ancho: 170,0 micras; extremidades: primera: longitud $=200,0$, segunda: 160,0 , tercera: 150,0 micras y cuarta: 77,0 micras; cabeza: longitud desde la base del capítulo: 100,0; ancho: 70,0 y gnathosoma: 25,0 micras y artejos: extremidad uno: uno: 17,0 , dos: 53,0 , tres: 23,0, cuatro: 44,0 y cinco: 58,0 micras (Figuras 9 y 10 ). 


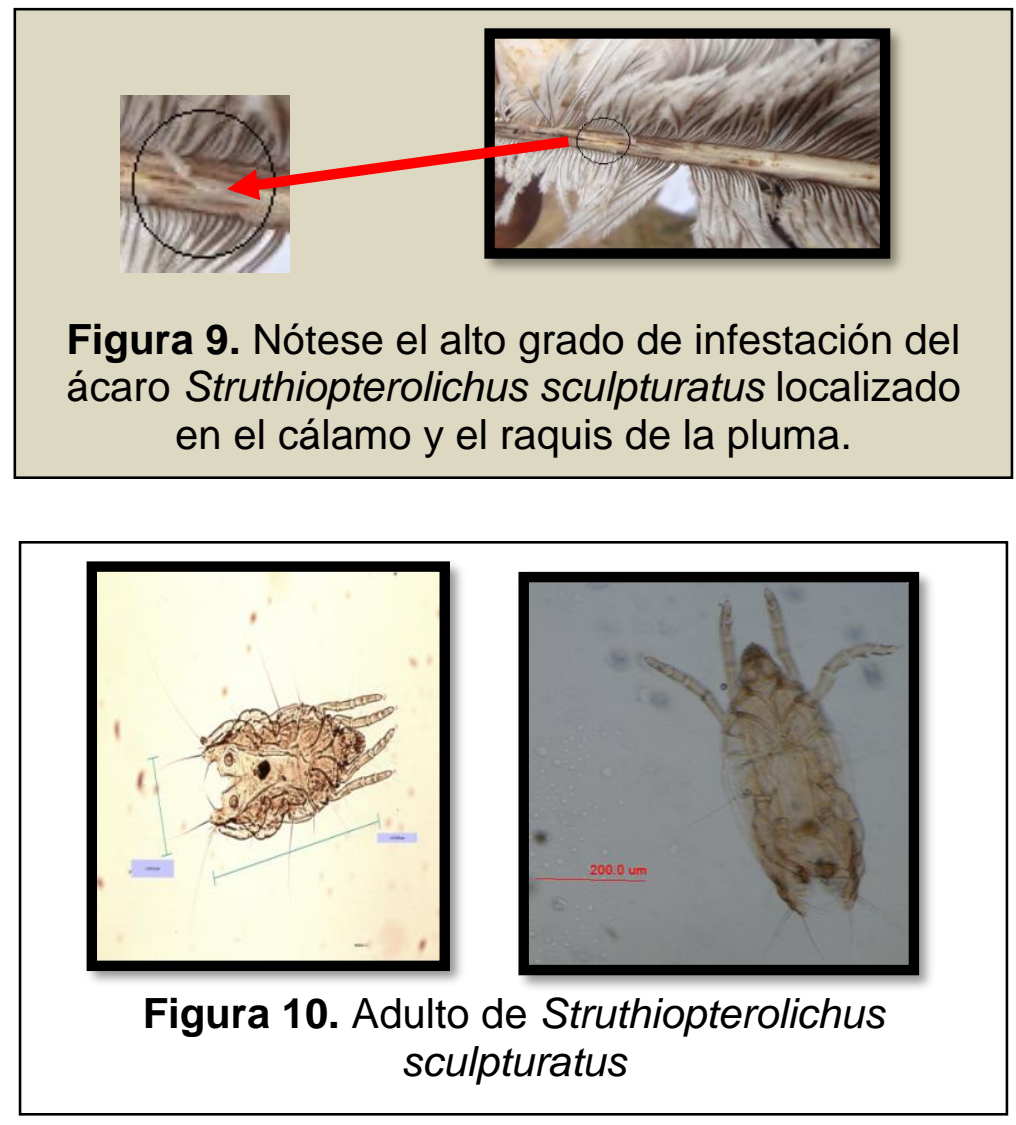

\section{REFERENCIAS BIBLIOGRÁFICAS}

1. Basso W, Venturini L. Risso M. Comparación de técnicas parasitológicas para el examen de heces de perro. Parasitol. día [online]. 1998:22 (1-2): 52-56.

2. Figueiroa L. De Freitas J. Oliveira B, Dowell M, Cavalcanti A. Soares A. et al. Parásitos gastrointestinales de aves silvestres en cautiverio en el estado de Pernambuco, Brasil. Parasitol. latinoam. [online]. 2002:.57, (1-2): 50-54.

3. Jiménez M. 2003. EL avestruz [consultado 25 de agosto 2011]. Disponible En: http://www.damisela.com/zoo/ave/ratities/avestruz/index.htm.

4. Lozada, H. Parasitología interna en animales domésticos. Compilación bibliográfica. Universidad de los Llanos. 2008, 82. Sp.

5. Margolis L, Esch W, Holmes J, Kuris A, Schad, G. The use of ecological terms in Parasitology (report of an ad hoc committee of the American Society of Parasitologists). J Parasitol.1982: 68(1): 131-133.

6. Negro, D. Avestruz rebaño de salud. Semin. Aves exóticas para mascotas, 2001:10:117-130.

7. Quiape A, Chavez V, Casas A et al. Prevalencia de Trypanosoma vivax en bovinos de cuatro distritos de la provincia de Coronel Portillo, Ucayali. Rev. investig. vet. Perú. [online]. 2003:14 (2): 161-165 [consultado 25 Agosto 2011].

Disponible

en: http://www.scielo.org.pe/scielo.php?script=sci arttext\&pid=S1609-

8. Rodríguez P, Sánchez A, Sacristán C. Libyostrongylus douglassi en la producción de avestruces. RCCV. 2007: I. (2): 544-548. 